\title{
De Novo mutations causing shwachman-diamond syndrome and a founder mutation in SBDS in the french canadian population
}

\begin{abstract}
We summarize the molecular findings in a patient cohort with Shwachman-Diamond syndrome that underwent genetic diagnostic testing. We could confirm a molecular diagnosis in 81 individuals. Our data is consistent with previous findings that the most common mutations in $S B D S$ are recurrent gene conversion mutations in exon 2, c. $258+2 \mathrm{~T}>\mathrm{C}$ or c.183_184delinsCT. The patients diagnosed either had two recurrent mutations (78\%), or one recurrent mutation and a rare family-specific mutation $(22 \%)$. We identified six unrelated individuals with SDS of French Canadian decent with the c.120del (p.Arg39fsX) mutation. Molecular analysis revealed that this mutation occurs with a founder haplotype. The opposing $S B D S$ mutation present in these individuals was the common c. $258+2 \mathrm{~T}>\mathrm{C}$ mutation, which was on a different haplotype in all five families. In addition, we estimated that approximately $9 \%$ (5 out of a subset of 54 patients with parent information) of the SDS individuals had a de novo mutation on one allele and an inherited mutation on the other allele. The de novo mutations were either recurrent gene conversion mutations $(\mathrm{n}=3)$ or rare point mutations $(\mathrm{n}=2)$. The occurrence of de novo mutation requires that parental carrier testing be performed for accurate familial risk assessment.
\end{abstract}

Keywords: shwachman-diamond syndrome, Sbds, De novo mutation, genetic testing
Volume I Issue 2 - 2014

\author{
Leslie Steele,' Johanna M Rommens, ${ }^{2,3}$ Tracy \\ Stockley, ${ }^{1,4}$ Berivan Baskin, ${ }^{5,6}$ Peter N Ray ${ }^{1,2,3,5}$ \\ 'Division of Molecular Genetics, The Hospital for Sick Children, \\ Canada \\ ${ }^{2}$ Department of Molecular Genetics, University of Toronto, \\ Canada \\ ${ }^{3}$ Program in Genetics and Genome Biology, The Hospital for \\ Sick Children, Canada \\ ${ }^{4}$ Department of Laboratory Medicine and Pathobiology, \\ University of Toronto, Canada \\ ${ }^{5}$ The Centre for Applied Genomics, The Hospital for Sick \\ Children, Canada \\ ${ }^{6}$ Department of Immunology, Genetics and Pathology, Uppsala \\ University, Sweden
}

Correspondence: Berivan Baskin, Department of Immunology, Genetics and Pathology, The Rudbeck Laboratory, Uppsala University, Uppsala, Sweden, Tel +46 7089664 06, Fax +46 18

5540 25, Email berivan.baskin@akademiska.se

\section{Abbreviations: SDS, shwachman-diamond syndrome \\ Introduction}

Shwachman-Diamond syndrome (SDS; OMIM260400) is a rare autosomal recessive disorder characterized with exocrine pancreatic dysfunction, bone marrow failure, neurocognitive anomalies and skeletal abnormalities. ${ }^{1}$ Patients frequently present with failure to thrive, susceptibility to infections and short stature. SDS is estimated to affect 1 in 100,000 individuals. ${ }^{2}$ SDS is caused by mutations in $S B D S$ (OMIM607444) located on chromosome 7q11and consists of 5 exons and spans $9 \mathrm{~kb} .{ }^{3}$ The majority of mutations in $S B D S$ are caused by gene conversion between $S B D S$ and its unprocessed pseudogene SBDSP1 located 5.85 MB distally. ${ }^{3}$ The pseudogene transcript is $97 \%$ identical to $S B D S$ and contains deletions and nucleotide changes that disrupt coding potential. Over $90 \%$ of individuals with SDS have at least one mutated $S B D S$ allele due to gene conversion. The three most common mutations; c. 183 184delinsCT, c.258+2T $>$ C, c.(183_184delinsCT; $258+2 \mathrm{~T}>\mathrm{C})$, arise from gene conversion between exon 2 of $S B D S$ and $S B D S P$ and account for approximately $80 \%$ of disease alleles in SDS. ${ }^{4-6}$ Remaining mutations in $S B D S$ are point mutations; missense, splice, nonsense and indels and can be located in any of the five exons. ${ }^{6,7}$ Some of these mutations have also been found to be the result of gene conversion. We describe the molecular findings in a patient cohort with SDS that were screened for mutations in SBDS. We could confirm a molecular diagnosis in 81 individuals. We determined that approximately $9 \%$ (5 out of 54) of unrelated individuals with SDS have a de novo mutation on one allele. In addition we identified a founder mutation, c.120del (p.Arg39fsX), in six unrelated individuals with SDS in the French Canadian population.

\section{Materials and methods}

\section{Patients}

Patients analyzed for $S B D S$ mutations were those suspected to have SDS and referred to the molecular genetics laboratory at the Hospital for Sick Children for genetic testing since 2004.

\section{SBDS sequencing}

DNA from peripheral blood samples were analyzed in the genetic diagnostic laboratory at The Hospital for Sick Children. All five exons and flanking intron sequences of SBDS (NM_016038.2) were sequenced both in forward and reverse direction. Sanger sequencing was performed according to standard protocols using BigDye terminator v1.1 (Life Technologies) and sequencing products were separated on an ABI model 3730 Capillary Sequencer (Life Technologies) and analyzed using SeqPilot software (JSI Medical Systems). Sequence nomenclature is based on the recommendations of HGVS (www.hgvs.org/mutnomen/).

\section{Haplotype analysis}

The STR markers (D7S499, D7S494, D7S2429, D7S502 and D7S482) and SNPs (rs1061695 and rs73151675) were used to determine the haplotype harboring the c.120del mutation.

\section{Results and discussion}

We performed diagnostic testing of 500 unrelated patients at various stages of investigation or suspicion of an SDS diagnosis in our molecular diagnostic laboratory over a ten-year span. We could confirm 
a molecular diagnosis in 81 probands (Table 1). All 81 individuals harbored at least one of the three most common gene conversion mutations, and the diagnosed patients either had two recurrent mutations $(78 \%)$, or one recurrent mutation and a rare family-specific mutation (22\%), consistent with previous reports. ${ }^{3,5}$ Parental samples were available for 54 patients to determine their carrier status. In five out of the 54 patients we could determine that one of the mutated alleles was de novo. To exclude sample mix-up and non-paternity/ maternity, DNA identity studies were performed which confirmed correct parentage within the families. Three of de novo mutations were the common gene conversion mutations, c.183_184delinsCT $(n=2)$ and $c .258+2 T>C(n=1)$, the fourth was a previously reported splice-site mutation c. $624+1 \mathrm{G}>\mathrm{A}$, and the fifth one was a previously unreported missense mutation, c.170T $>C$ (p.Phe57Ser). In four of the families the de novo mutations were detected in the probands and in the fifth family the mutation was found to be de novo in the proband's mother (Figure 1). The fifth proband was a compound heterozygote for the common mutations c. 183 184delinsCT and c.258+2T>C. The proband's mother carried the c.183_184delinsCT SBDS mutation while the proband's father and paternal grandfather carried the c. $258+2 \mathrm{~T}>\mathrm{C}$ SBDS mutation. Neither maternal grandparent carried the c.183_184TA $>$ CT $S B D S$ mutation indicating that the proband inherited a mutation from the mother that likely arose from a de novo gene conversion event in the grandparental generation between SBDS and SBDSP. During clinical testing of the referred SDS patients it was noted that six unrelated probands were compound heterozygotes with the same rare $S B D S$ mutation, c.120del, in exon 1, on one allele.

Table I Mutations identified in 8I unrelated probands with SDS

\begin{tabular}{|c|c|c|c|c|c|c|}
\hline \multirow{2}{*}{$\begin{array}{l}\text { Allele I } \\
\text { Mutation }\end{array}$} & \multirow[b]{2}{*}{ Reference } & \multirow[b]{2}{*}{$\begin{array}{l}\text { Inherited/ } \\
\text { de novo }\end{array}$} & \multicolumn{2}{|l|}{ Allele 2} & \multirow[b]{2}{*}{$\begin{array}{l}\text { Inherited/ } \\
\text { de novo }\end{array}$} & \multirow[b]{2}{*}{$\begin{array}{l}\text { Number } \\
\text { of patients }\end{array}$} \\
\hline & & & Mutation & Reference & & \\
\hline c.183_I84delinsCT (p.Lys62X) & Boocock et al. ${ }^{3}$ & unknown & c. $258+2 T>C($ p.Cys84fsX) & Boocock et al. ${ }^{3}$ & unknown & 23 \\
\hline c.183_I84delinsCT (p.Lys62X) & Boocock et al. ${ }^{3}$ & inherited & c. $258+2 T>C($ p.Cys84fsX) & Boocock et al. ${ }^{3}$ & inherited & 33 \\
\hline c.183_I84delinsCT (p.Lys62X) & Boocock et al. ${ }^{3}$ & de novo & c.258+2T>C(p.Cys84fsX) & Boocock et al. ${ }^{3}$ & inherited & 2 \\
\hline c.183_I84delinsCT (p.Lys62X) & Boocock et al. ${ }^{3}$ & inherited & c. $258+2 T>C(p . C y s 84 f s X)$ & Boocock et al. ${ }^{3}$ & de novo & I \\
\hline $\begin{array}{l}\text { c. } 183 \text { I84delinsCT; c.258+2T>C } \\
\text { (p.Lys62X;Cys84fsX) }\end{array}$ & Boocock et al. ${ }^{3}$ & inherited & c. $258+2 T>C($ p.Cys84fsX) & Boocock et al. ${ }^{3}$ & inherited & 4 \\
\hline c. $258+2 T>C(p . C y s 84 f s X)$ & Boocock et al. ${ }^{3}$ & unknown & c. $258+2 T>C($ p.Cys84fsX) & Boocock et al. ${ }^{3}$ & unknown & 2 \\
\hline c. $258+2 T>C(p . C y s 84 f s X)$ & Boocock et al. ${ }^{3}$ & inherited & c.120del (p.Arg39fsX) & Boocock et al. ${ }^{3}$ & inherited & 5 \\
\hline c. $258+2 T>C(p . C y s 84 f s X)$ & Boocock et al. $^{3}$ & unknown & c.120del (p.Arg39fsX) & Boocock et al. ${ }^{3}$ & unknown & I \\
\hline c. $258+2 T>C$ (p.Cys84fsX) & Boocock et al. ${ }^{3}$ & inherited & c. $38 \mathrm{C}>\mathrm{A}$ (p.Thr|3Asn) & Boocock et al. ${ }^{3}$ & inherited & I \\
\hline c. $258+2 T>C$ (p.Cys84fsX) & Boocock et al. ${ }^{3}$ & unknown & c. |29-| G>A (r.spl) & Donadieu et al. ${ }^{5}$ & inherited & I \\
\hline c. $258+2 T>C(p . C y s 84 f s X)$ & Boocock et al. ${ }^{3}$ & inherited & c. I70T>C (p.Phe57Ser) & This report & de novo & I \\
\hline c. $258+2 T>C(p . C y s 84 f s X)$ & Boocock et al. ${ }^{3}$ & inherited & c.4I0T>C (p.Met|37Arg) & This report & Inherited & I \\
\hline c. $258+2 T>C(p . C y s 84 f s X)$ & Boocock et al. ${ }^{3}$ & unknown & c.428C>T (p.Ser I43Leu) & This report & unknown & I \\
\hline c. $258+2 T>C(p . C y s 84 f s X)$ & Boocock et al. ${ }^{3}$ & unknown & c. $458 A>G$ (p.Gln I 53Arg) & Shammas et al. ${ }^{10}$ & inherited & I \\
\hline c. $258+2 T>C$ (p.Cys84fsX) & Boocock et al. ${ }^{3}$ & inherited & c. $624+$ IG $>A($ r.spl) & Tsangaris et al. ${ }^{\prime \prime}$ & de novo & I \\
\hline c. $258+2 T>C(p . C y s 84 f s X)$ & Boocock et al. ${ }^{3}$ & unknown & $\begin{array}{l}\text { c.650_65I delinsCT } \\
\text { (p.Phe2I7Ser) }\end{array}$ & This report & inherited & I \\
\hline
\end{tabular}




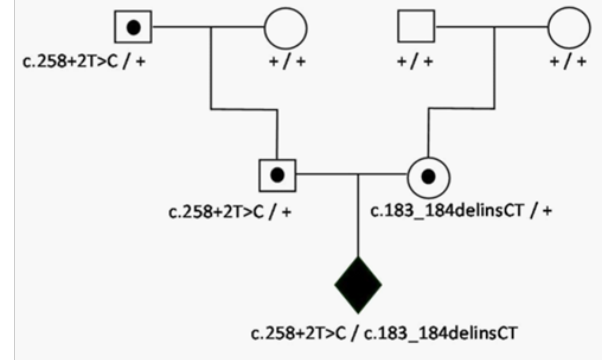

Figure I A de novo gene conversion mutation in a family with SDS. The proband was compound heterozygous for two common SBDS mutations (c. I83 I84delinsCT/c.258+2T>C). The proband's mother carried the c.183_I84delinsCT mutation while the proband's father carried the c. $258+2 T>C$ mutation. The paternal grandfather was the carrier of the c. $258+2 T>C$ mutation while neither maternal grandparent carried the c. I83_I84delinsCT mutation. This indicates that c. 183 184delinsCT was a de novo gene conversion event that was first evident in the proband's mother.
Further investigation indicated that all six probands were of French Canadian descent. That this mutation was not reported in a study of 102 French patients suggested that c.120del was a founder mutation arising as a de novo event after the migration of the French population Canada. To determine if this was the case we performed haplotype analysis using STR markers and SNPs within and flanking $S B D S$ on five of the probands and their family members (Figure 2). The haplotype analysis indicated a shared region in all five families, extending from $\sim 9.0 \mathrm{cM}$ proximal to at least $0.8 \mathrm{cM}$ distal of $S B D S$. Data on STR markers (D7S494 and D7S2429) and SNPs (rs1061695 and rs73151675) supports a common haplotype for the c.120del SBDS mutation in the French Canadian population. The chromosome region indicated in red shows the shared haplotype in all five families. The second $S B D S$ allele present in these individuals was the common c. $258+2 \mathrm{~T}>\mathrm{C}$ mutation that occurred on a different haplotypes in all five families. ${ }^{8}$

A

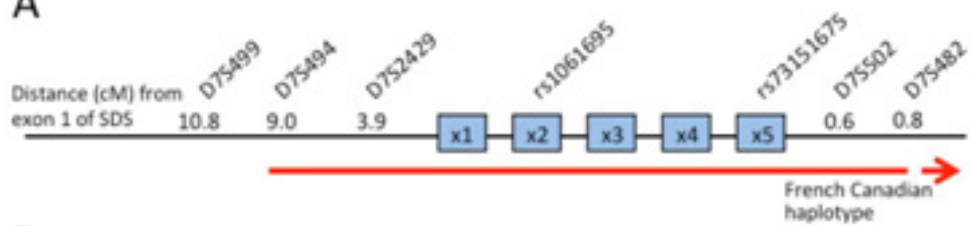

B
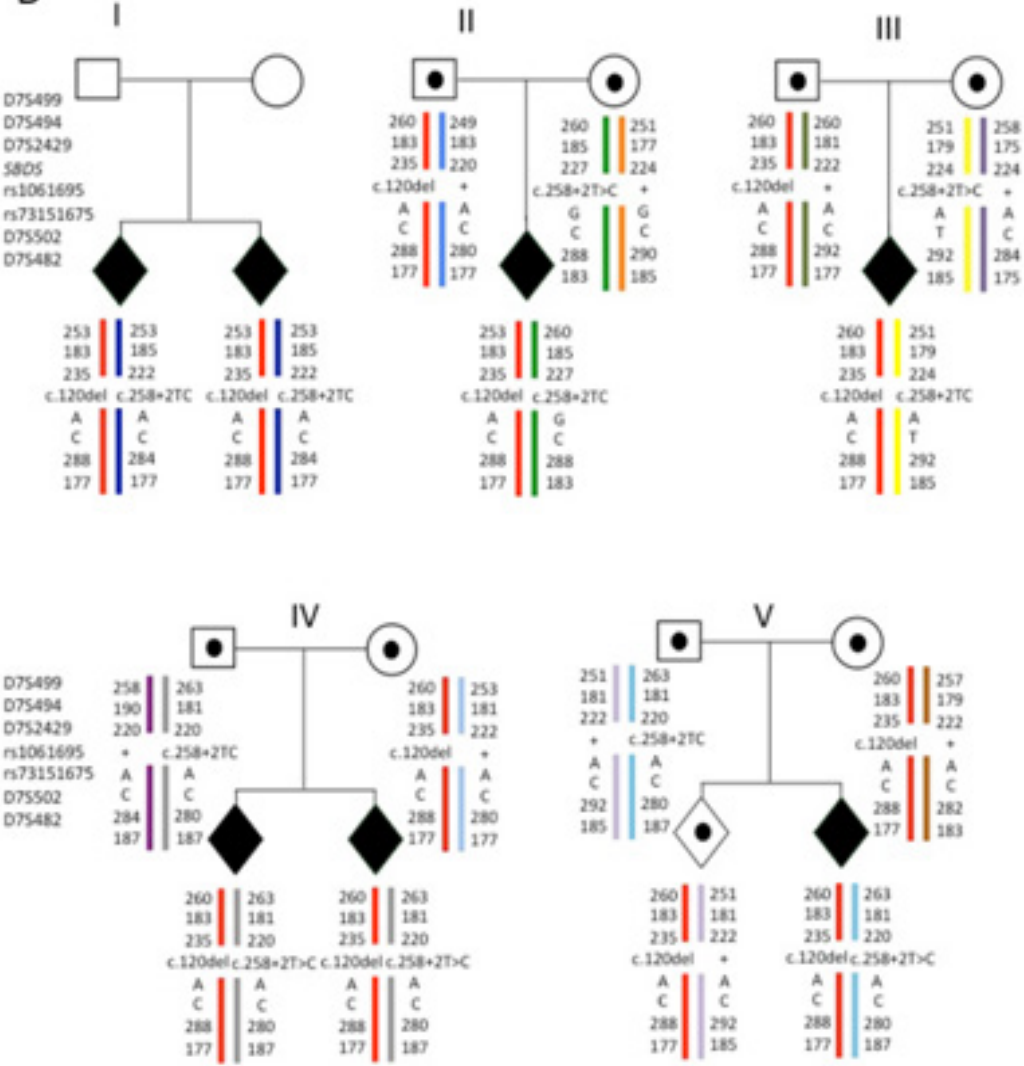

Figure $2 \mathrm{~A}$ founder mutation in SBDS in the French Canadian population.

A) A schematic map of the location of the STR markers and SNPs used in the haplotype analysis B) Five unrelated probands (family I-V) were compound heterozygotes for c.258+2T >C, and c. I20del. The five probands were all of French Canadian descent. Haplotype analysis using markers flanking SBDS indicated a shared region in all five families, extending from $\sim 9.0 \mathrm{Mb}$ proximal to $\geq 0.6 \mathrm{Mb}$ distal of SBDS.The STR marker (D7S494 and D7S2429) and SNP (rs 1061695 and rs73151675) alleles are consistent with a common haplotype for the c.I20del SBDS mutation in the French Canadian population. The region indicated in red shows the shared haplotype region in all five families. 


\section{Conclusion}

While the exact rate of de novo mutations remains unknown recent data from whole genome/whole exome sequencing of disease trio cohorts suggest they may play a more prominent role in disease than previously thought. ${ }^{9}$ While the most common $S B D S$ mutations are the result of gene conversion due to the presence of the unprocessed pseudogene copy (SBDSP1), the occurrence of five de novo mutations in 54 families studied indicates that de novo mutations have a significant impact on the pathogenesis of SDS. Moreover, a new founder mutation in the French Canadian population appears to be due to a relatively recent de novo event as this mutation is not described in France. Thus de novo mutations need to be considered when conducting molecular diagnosis of this disease. Analysis of parental samples to confirm that a $S B D S$ mutation found in a patient is in fact inherited from a parent is required in order to accurately estimate the recurrence risk of the disease in subsequent pregnancies.

\section{Acknowledgements}

None.

\section{Conflict of interest}

Author declares that there is no conflict of interest.

\section{References}

1. Dror Y, Donadieu J, Koglmeier J, et al. Draft consensus guidelines for diagnosis and treatment of Shwachman-Diamond syndrome. Ann NY Acad Sci. 2011;1242:40-55.

2. Cipolli M. Shwachman-Diamond syndrome: clinical phenotypes. Pancreatology. 2001;1(5):543-548.
3. Boocock GR, Morrison JA, Popovic M, et al. Mutations in SBDS are associated with Shwachman-Diamond syndrome. Nat Genet. 2003;33(1):97-101.

4. Woloszynek JR, Rothbaum RJ, Rawls AS, et al. Mutations of the SBDS gene are present in most patients with Shwachman-Diamond syndrome. Blood. 2004;104(12):3588-3590.

5. Donadieu J, Fenneteau O, Beaupain B, et al. Classification of and risk factors for hematologic complications in a French national cohort of 102 patients with Shwachman-Diamond syndrome. Haematologica. 2012;97(9):1312-1319.

6. Nicolis E, Bonizzato A, Assael BM, et al. Identification of novel mutations in patients with Shwachman-Diamond syndrome. Hum Mutat. 2005;25(4):410.

7. Nakashima E, Mabuchi A, Makita Y, et al. Novel SBDS mutations caused by gene conversion in Japanese patients with Shwachman-Diamond syndrome. Hum Genet. 2004;114(4):345-348.

8. Hashmi SK, Allen C, Klaassen R, et al. Comparative analysis of Shwachman-Diamond syndrome to other inherited bone marrow failure syndromes and genotype-phenotype correlation. Clin Genet. 2011;79(5):448-458.

9. Veltman JA, Brunner HG. De novo mutations in human genetic disease. Nat Rev Genet. 2012;13(8):565-575.

10. Shammas C, Menne TF, Hilcenko C, et al. Structural and mutational analysis of the $S B D S$ protein family. Insight into the leukemiaassociated Shwachman-Diamond syndrome. $J$ Biol Chem. 2005;280(19):19221-19229.

11. Tsangaris E, Klaassen R, Fernandez CV, et al. Genetic analysis of inherited bone marrow failure syndromes from one prospective, comprehensive and population-based cohort and identification of novel mutations. J Med Genet. 2011;48(9):618-628. 